\title{
Anabases
}

ANABASES Traditions et réceptions de l'Antiquité

5 | 2007

Varia

\section{Francesco ZAMBON, Alessandro Grossato, Il mito della fenice in Oriente e in Occidente}

\section{Cristina Noacco}

\section{OpenEdition}

Journals

Édition électronique

URL : http://journals.openedition.org/anabases/3254

DOI : 10.4000/anabases.3254

ISSN : 2256-9421

Éditeur

E.R.A.S.M.E.

Édition imprimée

Date de publication : 1 mars 2007

Pagination : 275-277

ISSN : 1774-4296

Référence électronique

Cristina Noacco, «Francesco Zambon, Alessandro Grossato, // mito della fenice in Oriente e in Occidente », Anabases [En ligne], 5 | 2007, mis en ligne le 01 décembre 2011, consulté le 22 septembre 2020. URL : http://journals.openedition.org/anabases/3254 ; DOI : https://doi.org/10.4000/anabases.3254

Ce document a été généré automatiquement le 22 septembre 2020.

(c) Anabases 


\title{
Francesco ZAMBON, Alessandro GrossATo, Il mito della fenice in Oriente $e$ in Occidente
}

\author{
Cristina Noacco
}

\section{RÉFÉRENCE}

Francesco ZAMBON, Alessandro Grossato, Il mito della fenice in Oriente e in Occidente,

Venise, Marsilio, 2004, $173 \mathrm{p}$.

35 euros / ISBN 88-317-8614-8.

1 D'Orient en Occident, le foisonnement du mythe du phénix n'a pas cessé de vivifier et de nourrir son vol d'une symbolique simple mais à l'enjeu puissant : la victoire de la vie sur la mort.

2 Dans la première partie du volume ("Il mito della fenice in Occidente ", p. 15-81), Francesco Zambon retrace les origines du mythe dans la culture occidentale à partir de l'oiseau égyptien benu, symbole de création et de résurrection, qu'Hérodote décrit comme un oiseau solaire. Le mythe gréco-romain résulterait ainsi de la réélaboration d'une croyance cosmologique-religieuse égyptienne, identifiant le phénix avec le soleil. Mais c'est dans un texte latin, résumé par Pline (Naturalis historia, V), que cet oiseau apparait avec les caractéristiques de longévité (540 ans) et de renaissance (à partir de sa mort) qu'on lui attribue. De ses os naît tout d'abord un ver, puis un oiseau : le cycle de la vie s'accomplit par les métamorphoses du phénix : les flammes et les cendres, cœur du mythe, appartiennent à une autre tradition, datant de la fin du $\mathrm{I}^{\mathrm{er}}$ siècle, celle de Martial et de Stace. Le mythe, issu de la convergence de ces traditions, apparaitt au $\mathrm{I}^{\mathrm{er}}$ siècle comme pleinement développé et le terme de phénix renvoie désormais au monde grec (phoînix, du nom de l'arbre, la palme, qui l'abrite).

3 Pour les chrétiens, le retour régulier du phénix fait de cet oiseau sacré du soleil le symbole d'un grand renouvellement historique, la naissance du Christ, ainsi que 
l'emblème de la vérité eschatologique : la résurrection des corps. L'association de la renaissance de l'oiseau et de la Résurrection du Christ a été développée dans le premier bestiaire chrétien, rédigé entre le $\mathrm{II}^{\mathrm{e}}$ et le $\mathrm{III}^{\mathrm{e}}$ siècle à Alexandrie : le Physiologus graecus. Cette nouvelle interprétation s'impose sur les précédentes : le phénix devient alors l'un des symboles majeurs du Christ.

La symbolique du phénix trouve de nombreuses applications même en dehors du domaine chrétien. Les auteurs du Moyen Âge et, en particulier, les troubadours, développent une version laïque du mythe, qui s'appuie sur la comparaison entre l'oiseau et l'amant, qui brûle et se consume par amour. Mais le trouvère Chrétien de Troyes, qui exploite la symbolique du phénix dans son deuxième roman, Cligès, rédigé vers 1176, a plutôt recours à l'idée de résurrection qui est également contenue dans le mythe : par le biais d'une fausse mort, l'héroïne, au nom révélateur de Fenice, meurt à la vie qu'elle n'a pas choisie et peut ainsi renaître au vrai amour, celui qu'elle nourrit pour Cligès. D'autres exemples de la fortune du motif dans le domaine laïque médiéval sont présents dans un chapitre du Bestiaire d'amours, du 1250 environ, de Richard de Fournival et, en Italie, dans le III livre de l'Acerba de Cecco d'Ascoli, où l'oiseau devient le symbole de la Femme céleste, la divine Sapience ou Intelligence. Ici, et encore plus chez Dante et Pétrarque, apparaît un thème destiné à une grande fortune dans la lyrique amoureuse italienne : la mort de la femme aimée. Pour Pétrarque, au terme de cette évolution, la femme-phénix meurt à sa vie réelle pour renaître comme sujet poétique.

5 À la Renaissance et pendant la période baroque, le phénix occupe une place de relief dans le courant hermétique-alchimique, où il est doublement interprété : d'un côté, il représente la Nature parfaite telle qu'elle est issue des mains de son Créateur (et, en ce sens, il est considéré comme la matière première de l'(Euvre), de l'autre, il illustre le résultat du processus alchimique tout entier: la Pierre philosophale. Pour les auteurs de l'époque baroque, le mythe du phénix est l'une des expressions les plus accomplies de l'union des contraires, si chère aux poètes de cette période.

6 L'oiseau mythique devient enfin, au XVIII ${ }^{\mathrm{e}}$ siècle, la métaphore de Venise, célébrée comme l'héritière de la Rome ancienne, malgré sa décadence politique et militaire et il va jusqu'à donner son nom au célèbre théâtre vénitien, plusieurs fois détruit par le feu et reconstruit.

7 Dans la deuxième partie du volume ("Il mito della fenice in Oriente", p. 83-173), Alessandro Grossato interroge la symbolique du phénix en Orient, dont il reconnaît cinq traditions principales et qu'il détaille à partir d'une caractéristique récurrente : la lumière, car cet oiseau mythique représente avant tout, comme pour les auteurs de l'Antiquité occidentale, le soleil.

8 Le Judaïsme compte au moins trois phénix : Ziz, Malham et Hol. L'oiseau géant Ziz a été placé par Dieu à la tête du royaume des airs (de même que Béhémot et Léviathan régissent respectivement la terre et l'eau). Le phénix nommé Malham est un symbole d'immortalité : ayant refusé de partager avec Ève le fruit défendu, il aurait vécu au Paradis terrestre jusqu'à la fin du monde. Le troisième phénix de la tradition juive, Hol, est décrit dans le Livre des secrets d'Énoch comme très semblable au Simurgh iranien : il a les pattes et la queue de lion, la tête de crocodile.

9 Dans l'ancienne culture soufi, l'oiseau mythique Simurgh est un emblème à la fois de royauté et de spiritualité. Parmi les textes les plus importants qui le mettent en lumière, cités par l'auteur, Le langage des oiseaux, de Farîd ad-dîn 'AttQr (1150-1220), en 
relate la quête de la part de toutes les espèces d'oiseaux. Cette quête est celle de chaque homme qui, doté d'un esprit immortel, donc d'essence divine (le phénix), cherche à rejoindre Dieu à travers un vol intérieur et chamanique, par le biais de la contemplation initiatique.

Dans la tradition islamique, véritable creuset de cultures, le phénix le plus ancien est l'«Anka », représenté sous forme de héron, comme le benu égyptien, ce qui a porté les auteurs grecs et latins à croire que cet oiseau était d'origine arabe. La diffusion de l'Islam a entraîné l'assimilation de l'« Anka » au Simurgh iranien et, d'après certains, au Garuda, l'oiseau solaire indien.

Le Garuda, équivalent indien du phénix, constitue la synthèse de toutes les divinités du panthéon indu, traditionnellement décrites comme semblables aux oiseaux, tandis que leurs adversaires sont figurés par des serpents. Le phénix se trouve donc au centre de la représentation du combat éternel entre le démoniaque et le divin.

A. Grossato considère que l'origine du phénix est à situer en Chine, où, autour du vIII siècle avant notre ère, sa symbolique solaire était intimement liée au rite ancestral du vol chamanique. À la différence des manifestations occidentales, les phénix chinois apparaissent souvent en couple et représentent la dualité cosmique : le yin et le yang. De même, l'on rencontre le phénix double, mâle (Hó) et femelle (ó), au Japon. Il est alors symbole, outre du soleil, de la fidélité et de l'obéissance.

Enfin, l'auteur souligne le fil conducteur qui relie toutes les manifestations du phénix dans les traditions orientales : le parallèle entre le vol du phénix et le vol ascensionnel du chaman, lors de sa troisième et dernière métamorphose.

14 Le livre que Francesco Zambon et Alessandro Grossato ont consacré au mythe du phénix en Orient et en Occident est un ouvrage qui présente des caractères à la fois d'érudition et de vulgarisation: sa prose claire et fluide permet à un large public d'accéder à des traditions souvent très complexes; ses renvois à des notes d'explication ou d'approfondissement nourrissent la curiosité des lecteurs les plus exigeants.

Il s'agit sans aucun doute d'un exemple remarquable d'étude thématique qui pourrait donner naissance à une collection entièrement consacrée aux animaux mythologiques. On souhaite que ce livre, à l'instar du premier ouvrage d'Alessandro Grossato sur la symbolique (Le livre des Symboles. Les métamorphoses de l'humain entre l'Orient et l'Occident, Milan-Paris, Mondadori-Éd. du Rocher, Préface de Gilbert Durand, 2000 [1 $1^{\text {re }}$ éd. Milan, Mondadori, 1999]), puisse être traduit dans les plus brefs délais en langue française, permettant ainsi au public francophone d'accéder à son tour au patrimoine culturel de l'un des mythes les plus répandus en Orient et en Occident.

\section{AUTEURS}

\section{CRISTINA NOACCO}

Université de Toulouse II - Le Mirail

cnoacco@yahoo.fr 